\title{
Use of Amniotic Membrane in MMC-Augmented Trabeculectomy: A Retrospective Comparative Study
}

\author{
Joana Roque (iD' \\ Fernando Trancoso Vaz (D) \\ Rita Basto' \\ Susana Henriques' \\ Ana Sofia Lopes' \\ Diana Silva' \\ Jorge Santos $\mathbb{D D}^{2}$ \\ Graça Pires' \\ Maria Lisboa' \\ Isabel Prieto (iD) \\ 'Ophthalmology Department, Prof. \\ Doutor Fernando Fonseca Hospital, \\ Amadora, Lisbon, Portugal; ${ }^{2}$ Department \\ of Mathematics ECT, CIMA IIFA Évora \\ University, Évora, Portugal
}

Purpose: Amniotic membrane transplantation (AMT) has shown promising results as an antifibrotic agent in trabeculectomy. We aimed to evaluate the additional effect of AMT in MMC-augmented trabeculectomy.

Patients and Methods: This retrospective study analyzed the results of the first 12 postoperative months of glaucomatous eyes submitted to Moorfields Safer Surgery Trabeculectomy with MMC alone (non-AMT group) compared to MMC and AMT (AMT group). Both groups were compared in terms of intraocular pressure (IOP), number of antihypertensive medications and need for surgical reinterventions. Absolute and relative success rates 12 months after surgery were defined as IOP $<18 \mathrm{mmHg}$, without and with the use of antihypertensive medications, respectively.

Results: The analysis included 51 eyes of 45 glaucoma patients (29 eyes in the non-AMT group and 22 in the AMT group). Mean IOP decreased from $24.72 \pm 5.11 \mathrm{mmHg}$ and 26.86 $\pm 10.62 \mathrm{mmHg}$ preoperatively in non-AMT and AMT groups to $12.86 \pm 4.22 \mathrm{mmHg}$ and 12.60 $\pm 4.43 \mathrm{mmHg}$, respectively, at 12 months $(\mathrm{p}=0.84)$. Postoperative number of medications decreased significantly in both groups. Absolute success was seen in $71 \%$ of non-AMT eyes and $55 \%$ of AMT eyes $(\mathrm{p}=0.46)$, whereas relative success was obtained in $14 \%$ and $30 \%$, respectively ( $p=0.55$ ). Reinterventions were needed in $28 \%$ of the eyes ( 11 bleb injection/ needling and 4 Ahmed tube implantation) in the non-AMT group and in $27 \%$ of the AMT group (10 bleb injection/needling and 1 Ahmed tube implantation) ( $p=0.89)$.

Conclusion: Trabeculectomy combined with MMC and AMT did not show better results than trabeculectomy with MMC alone.

Keywords: trabeculectomy, glaucoma, mitomycin C, amniotic membrane, antifibrotics, scarring

\section{Introduction}

Trabeculectomy is the gold standard procedure in glaucoma surgery worldwide. ${ }^{1}$ It creates a drainage pathway between the anterior chamber and the subconjunctival space leading to a subconjunctival reservoir for the aqueous humor, referred to as the filtering bleb.

The technique was first described in 1968 by Cairns. ${ }^{2}$ Since then, several modifications to this procedure, such as the "Moorfields Safer Surgery System" (MSS Trab) have improved surgical safety, predictability and outcomes. ${ }^{3}$ MSS Trab includes modifications such as a fornix-based conjunctival dissection, the use of an anterior chamber stabilizer, the use of adjustable/removable sutures and a wider
Correspondence: Joana Roque Ophthalmology Department, Prof. Doutor Fernando Fonseca Hospital, Amadora, Lisbon, ICI 9 2720-276, Portugal

Tel +351932828099

Email joana.roque@hff.min-saude.pt 
surface area for antifibrotics application. Thereby, current procedures aim to achieve larger and more diffuse filtering blebs, less prone to long-term failure, leakage and infectious complications.

Nevertheless, the wound healing process that occurs after surgery still leads to an excessive fibrosis at the subconjunctival space. This represents the ultimate reason for bleb failure and uncontrolled IOP after surgery. Therefore, glaucoma surgeons use antifibrotic agents such as Mitomycin C (MMC) and 5-Fluorouracil (5-FU) in a great proportion of the eyes undergoing trabeculectomy, according to the individuals' risk of bleb failure. ${ }^{3}$ None of these antifibrotic agents has, however, completely superseded conjunctival scarring. On the other hand, these agents are associated with several short- and long-term complications, such as bleb avascularity, thinning of the conjunctiva, hypotony, and increased risk of endophthalmitis, hence the need for more specific modulators of the wound-healing pathway. ${ }^{4}$

Newer adjunctive agents have been investigated in order to increase bleb survival. These include antivascular endothelial growth factor (VEGF) agents, ${ }^{5,6}$ space occupying components such as the Ologen ${ }^{\circledR}$ implant, ${ }^{7}$ and also amniotic membrane transplantation (AMT). Although none of these has clearly supplanted MMC-augmented trabeculectomy, amniotic membrane has been increasingly investigated in glaucoma surgery, since it was first described in $1998 .{ }^{8}$ Human amniotic membrane is widely known for its anti-inflammatory, antifibrotic and antiangiogenic properties, distinctly acting at different levels of the wound healing process. It has shown promising results as a physiological bleb modulator in glaucoma filtering surgeries. ${ }^{9-11}$

There are some data suggesting a favorable effect of the combination of AMT with MMC-augmented trabeculectomy but it is still unclear if this should be a routine procedure. ${ }^{12-14}$ This study evaluated the results of glaucoma patients submitted to trabeculectomy with MMC compared to patients submitted to trabeculectomy with simultaneous use of MMC and amniotic membrane.

\section{Materials and Methods}

This retrospective comparative study analyzed the results of the first 12 postoperative months of glaucoma patients submitted to trabeculectomy at the Glaucoma Department of Prof. Doutor Fernando Fonseca Hospital, Amadora, Lisbon, Portugal, from January 2015 to May 2018. The study respected the tenets of the Declaration of Helsinki and was approved by the Prof. Doutor Fernando Fonseca Hospital Ethics Committee. A written informed consent was obtained from all patients in the study.

\section{Patients and Treatments}

Clinical data from 51 eyes of 45 glaucoma patients (openangle and angle-closure glaucoma) were retrospectively analyzed. Among them, 29 eyes were treated with MMCaugmented trabeculectomy (non-AMT group) and 22 eyes were treated with MMC-augmented trabeculectomy combined with amniotic membrane transplantation (AMT group). The latter were operated mainly since the beginning of 2017, once AMT was a more established procedure. We included only patients whose clinical records were complete. Patients who had had glaucoma surgery or any type of conjunctival surgery before were excluded, as patients with coexisting conjunctival disease.

\section{Surgical Methods}

All trabeculectomies were performed by the same two surgeons according to Moorfields Safer Surgery System. ${ }^{3}$ A fornix-based conjunctival flap was made on the upper margin of the temporal quadrant, along with an extended and careful subconjunctival dissection. A scleral flap of $4 \times 4 \mathrm{~mm}$ with $1 / 3$ thickness of sclera was cut $0.5 \mathrm{~mm}$ from the limbus. Polyvinyl alcohol sponges soaked in MMC were applied in the largest possible area of the subconjunctival space and under the scleral flap for 3 minutes, followed by a thorough washing with saline solution. MMC concentration was selected according to Moorfields/Florida "More Flow" regime, which stratifies patients into three levels of risk for trabeculectomy failure. ${ }^{3}$ In all cases, the $0.4 \mathrm{mg} / \mathrm{mL}$ concentration was preferred. Afterwards, using an anterior chamber maintainer, a standardized sclerostomy was performed with a $0.5 \mathrm{~mm}$ punch. After iridectomy, the scleral flap was sutured with two fixed sutures along with two adjustable/removable sutures. For patients in the AMT group, before suturing the conjunctiva, the previously defrosted human amniotic membrane was implanted above the scleral flap (Figure 1) with the stromal surface facing upwards (allowing the antifibrotic mediators to contact with the subconjunctival fibroblasts). The single-layered amniotic membrane was then fixed near the limbus with 2 sutures (10/ 0 nylon) and spread towards the fornix. The conjunctiva was sutured with two interrupted 6/0 Vicryl sutures at the ends of the conjunctival incision Postoperative management included application of topical dexamethasone phosphate 




Figure I Amniotic membrane above the scleral flap (stromal surface facing down).

$0.1 \% \mathrm{q} 4 \mathrm{~h}$ and ofloxacin $0.3 \% \mathrm{q} 6 \mathrm{~h}$, tapered over several weeks.

\section{Postoperative Follow-Up and Efficacy Evaluation}

Pre and postoperative IOP, number of antihypertensive medications, as well as need for surgical reinterventions (5-FU or MMC bleb injection/needling or glaucoma drainage device implantation) data were collected from the following follow-up visits: week 1 and months 1, 2, 3, 4, 5, 6, 8 and 12 .

Filtering bleb morphology/quality is a subjective characteristic difficult to assess retrospectively (large and diffuse, flat, cystic, avascular, etc.) (Figure 2). Thus, to evaluate the efficacy of the procedures, we defined success criteria. Absolute success was defined as IOP $<18 \mathrm{mmHg}$ without antihypertensive medications or the need for any type of

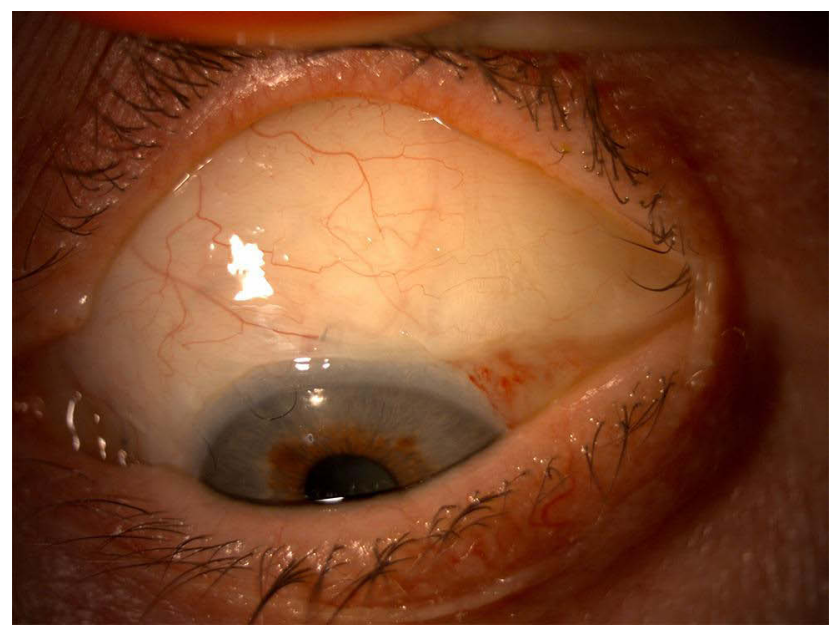

Figure $\mathbf{2}$ Large and diffuse filtration bleb registered in one of the eyes of the AMT group. reinterventions, at 12 months after operation. Relative success was defined as IOP $<18 \mathrm{mmHg}$ with the use of IOP-lowering medications at the same timepoint. We also analyzed the need and the timing for reinterventions in those eyes presenting with nonfunctioning blebs.

\section{Statistical Analysis}

The statistical analysis was performed by a statistician, using IBM $^{\circledR}$ SPSS $^{\circledR}$ Statistics. Continuous variables were analyzed using the independent Student's $t$-test and Welch's unequal variances $t$-test. The categorical variables were analyzed using the chi-square test or Fisher's exact test depending on sample size. A $p<0.05$ was considered significantly different.

\section{Results}

\section{Preoperative Baseline Characteristics}

Fifty-one glaucomatous eyes were analyzed in this study (29 eyes in the non-AMT group and 22 eyes in the AMT group). As shown in Table 1, demographics and preoperative baseline characteristics were similar between both groups. Overall mean preoperative IOP (95\% confidence interval) was $25.72 \pm 2.39 \mathrm{mmHg}$.

The study included eyes with both open angle and angle closure glaucoma: 23 eyes with primary open angle glaucoma (POAG), 14 eyes with primary angle closure glaucoma, 10 eyes with pseudoexfoliative glaucoma, 3 eyes with juvenile glaucoma and 1 eye with steroid-induced glaucoma.

We checked for the homogeneity of the data through both treatment groups, concerning age, gender, race, and glaucoma classification. At a significance level of 5\%, we cannot reject homogeneity.

\section{Postoperative IOP, Medications and Success Rates}

Follow-up time considered for the study was a minimum of 12 months for all patients. Mean postoperative IOP values in both groups are presented in Table 2. Figure 3 shows mean IOP evolution in both groups during the twelve-month period. Postoperative mean IOP in the AMT group was significantly lower $(p=0.02)$ than nonAMT group only at the first postoperative week (7.05 and $10.37 \mathrm{mmHg}$, respectively). On follow-up months 1, 3, 6, and 12, mean IOP was lower in the AMT group, however, without statistical significance.

Patients in both study groups used a mean of 4 hypotensive topical medications preoperatively, which decreased 
Table I Demographics and Preoperative Baseline Characteristics

\begin{tabular}{|c|c|c|c|}
\hline Parameter & Non-AMT Group $(n=29)$ & AMT Group $(n=22)$ & p-value \\
\hline Mean preoperative IOP \pm SD & $24.72 \pm 5.1 \mathrm{I} \mathrm{mmHg}$ & $26.86 \pm 10.62 \mathrm{mmHg}$ & $0.40^{\mathrm{a}}$ \\
\hline \multicolumn{4}{|l|}{ Age } \\
\hline Mean & 65 years & 64 years & $0.72^{\mathrm{b}}$ \\
\hline Range & $3|-8|$ years & $29-78$ years & \\
\hline Gender (F:M) & $16: 13$ & $6: 16$ & $0.08^{c}$ \\
\hline \multicolumn{4}{|l|}{ Race } \\
\hline White/Non-white & $24 / 5$ & $18 / 4$ & 1.00 \\
\hline Glaucoma classification & & & $0.85^{d}$ \\
\hline Primary open angle & 12 & II & \\
\hline Primary angle closure & 8 & 6 & \\
\hline Pseudoexfoliative & 6 & 4 & \\
\hline Juvenile & 2 & 1 & \\
\hline Steroid-induced & I & - & \\
\hline
\end{tabular}

Abbreviations: SD, standard deviation; F, female; M, male; a, Welch's unequal variances $t$-test; b, Student's $t$-test; c, Fisher's exact test; d, Pearson chi-square test.

significantly in both groups. Along the studied period, the number of IOP-lowering medications tended to be higher in the AMT group, with a significant difference at 6 months after surgery (1.83 vs 0.32) (Figure 4). Twelve months after surgery, mean number of medications was 0.61 in the non-AMT group and 1.4 in the AMT group ( $\mathrm{p}=0.06$ ).

Regarding success rates, absolute success was higher in the non-AMT group than in the AMT group, $71 \%$ and $55 \%$, respectively $(\mathrm{p}=0.46)$. Relative success rates were accomplished in $14 \%$ and in $30 \%$ of the eyes in the non-AMT and AMT groups, respectively ( $\mathrm{p}=0.55$ ). Disregarding the use of hypotensive medications, $86 \%$ of the eyes in the non-AMT group and $85 \%$ of the eyes in the AMT groups achieved IOP below the considered cutoff $(\mathrm{p}=0.63)$.

\section{Need for Reinterventions}

Surgical reinterventions were needed in 8 eyes $(28 \%)$ of the non-AMT group and in 6 eyes $(27 \%)$ of the AMT group $(p=0.89)$. Table 3 specifies the type of procedures performed

Table 2 Postoperative Mean IOP and Standard Deviations at Follow-Up Visits

\begin{tabular}{|l|l|l|l|}
\hline & $\begin{array}{l}\text { Non-AMT Group } \\
(\mathbf{m m H g})\end{array}$ & $\begin{array}{l}\text { AMT Group } \\
(\mathbf{m m H g})\end{array}$ & p-value \\
\hline I week & $10.37 \pm 5.47$ & $7.05 \pm 2.13$ & 0.02 \\
I month & $13.87 \pm 6.12$ & $12.57 \pm 7.35$ & 0.53 \\
3 months & $14.73 \pm 5.55$ & $14.33 \pm 4.79$ & 0.81 \\
6 months & $13.58 \pm 3.85$ & $12.07 \pm 3.91$ & 0.28 \\
I2 months & $12.86 \pm 4.22$ & $12.60 \pm 4.43$ & 0.84 \\
\hline
\end{tabular}

in each group. The eyes in the non-AMT group required 15 reinterventions: 11 5-FU bleb injections/needling and 4 surgeries for Ahmed tube implantation. The ones in the AMT group were submitted to 11 reinterventions: 35 -FU bleb injections/needling, $7 \mathrm{MMC}$ bleb injections/needling and 1 Ahmed tube implantation. We did not find statistical significance in the number of eyes that showed complete failure of trabeculectomy culminating in glaucoma drainage device (GDD) implantation (4/29 eyes in non-AMT group and 1/22 eyes in AMT group, $\mathrm{p}=0.27$ ). Mean time until first reintervention was also similar: 3.1 months in the non-AMT group and 2 months in the AMT group.

\section{Discussion}

The healing process at the filtration bleb is the main reason for trabeculectomy failure, thus wound healing modulation has been thoroughly investigated as the key element to improve trabeculectomy outcomes.

Human amniotic membrane is the innermost layer of the placenta. It serves as a valuable adjunct tool for ocular surgery because of its many beneficial features: it is transparent, lacks immunogenicity, and has been shown to have antiinflammatory, antifibrotic, antiangiogenic, and possibly antimicrobial properties. It is primarily used in the treatment of ocular surface disorders, serving as a substrate for epithelium to grow on. On the other hand, the stromal surface of amniotic membrane stands out for downregulating Tumor Growth Factor- $\beta$ signaling, thus reducing fibroblast production and myofibroblast differentiation, leading to decreased scar 


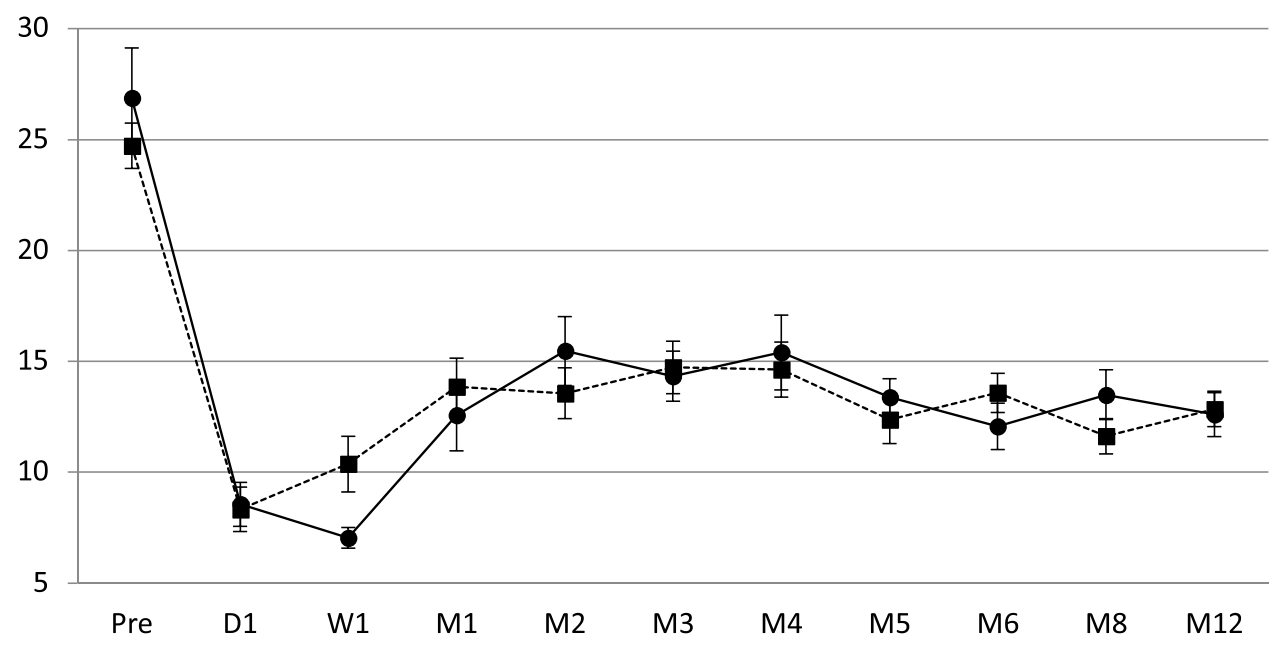

Figure 3 Preoperative and postoperative mean IOP during follow-up period. We present mean IOP values in each study group. The bars represent the standard error at each follow-up visit. Vertical axis - Mean IOP in each group; horizontal axis - preoperative and postoperative visits; solid line - non-AMT group; dashed line - AMT group.

formation. It also inhibits proinflammatory cytokines and inflammatory cell migration, resulting in a reduced inflammatory reaction and vascularization. ${ }^{15}$

Based on these properties, amniotic membrane has been proposed as an adjunct to trabeculectomy, in order to prevent healing in the subconjunctival space and to promote longliving blebs. Experimental rabbit studies have demonstrated that amniotic grafts at the trabeculectomy site lead to less fibroblast cell counts and better bleb function. ${ }^{16,17}$ Since Fujishima's study in $1998,{ }^{8}$ several clinical studies pointed to the favorable outcomes of addition of AMT to trabeculectomy without antifibrotics, ${ }^{9}$ however some of them lacking statistical significance. The randomized trial performed by Eliezer et al did not find a statistically significant IOP-lowering effect of a folded amniotic membrane graft in classic trabeculectomy. ${ }^{18}$ More recently, Stavrakas and his colleagues enrolled 59 eyes with POAG in a prospective randomized trial over 24 months comparing trabeculectomy without antimetabolites to trabeculectomy combined with AMT. The reduction of IOP was greater in the AMT group throughout the follow-up period but this difference was not statistically significant at any point. ${ }^{11}$

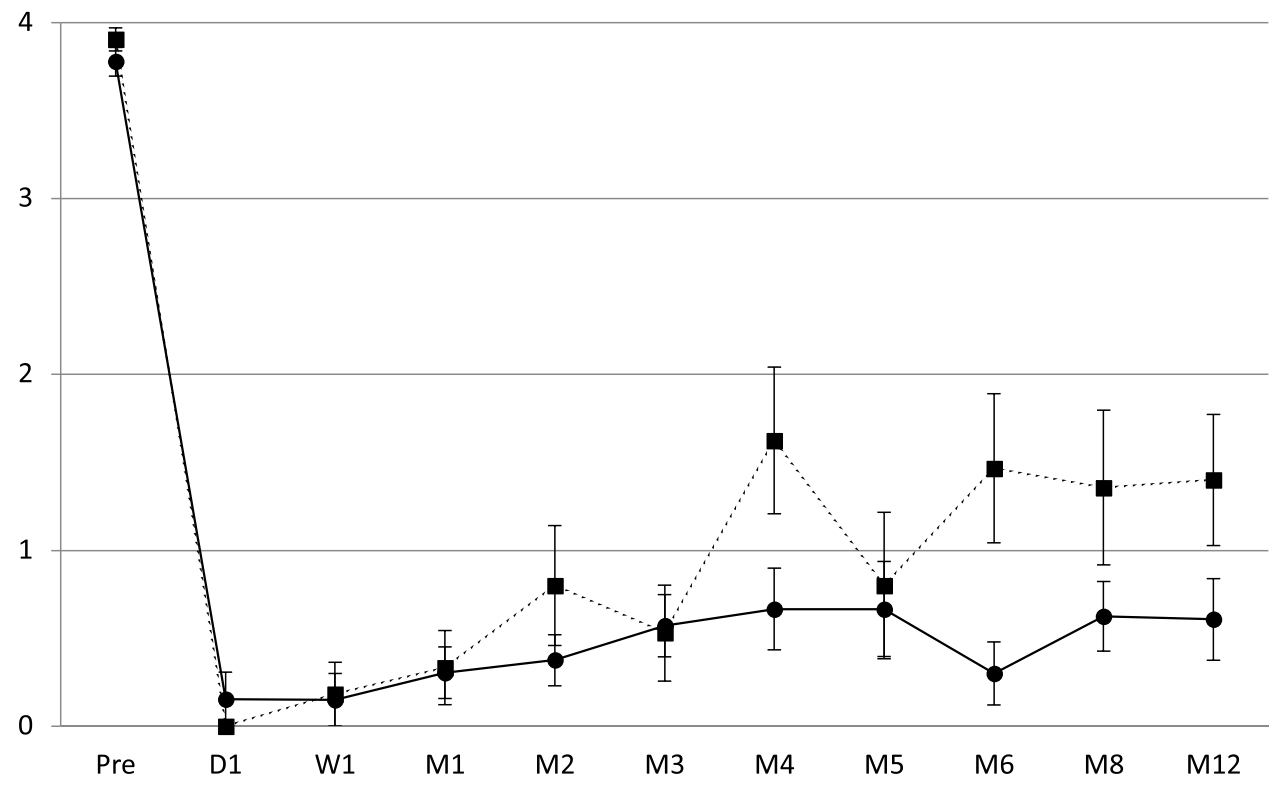

Figure 4 Preoperative and postoperative mean number of antihypertensive medications during follow-up period. Representation of the mean number of antihypertensive medications used in each group, along with bars relative to the standard error at each timepoint. Vertical axis - mean number of antihypertensive medications in each group; horizontal axis - preoperative and postoperative visits; solid line - non-AMT group; dashed line - AMT group. 
Table 3 Type of Reintervention Performed

\begin{tabular}{|l|l|l|}
\hline Type of Reintervention & $\begin{array}{l}\text { Non-AMT Group } \\
\mathbf{n}=\mathbf{2 9}\end{array}$ & $\begin{array}{l}\text { AMT Group } \\
\mathbf{n}=\mathbf{2 2}\end{array}$ \\
\hline $\begin{array}{l}\text { 5-FU bleb injection/needling } \\
\text { MMC bleb injection/needling }\end{array}$ & II (7 eyes) & None $(2$ eyes) \\
GDD implantation (Ahmed tube) & 4 (4 eyes) & 7 (5 eyes) \\
Total & 15 ( 1 eye $)$ \\
1 eyes $)$ & $(6$ eyes $)$ \\
\hline
\end{tabular}

Note: Some of the eyes subject to bleb injection/needling were subsequently submitted to a GDD surgery.

Abbreviation: GDD, glaucoma drainage device.

Other authors compared trabeculectomy with AMT versus trabeculectomy with antifibrotics. The randomized trial conducted by Khairy et al, involving 52 eyes with POAG, revealed that trabeculectomy with AMT had a comparable IOP-lowering effect but a reduced rate of postoperative complications after 24 months of follow-up compared to trabeculectomy with MMC. ${ }^{10}$

To the best of our knowledge, only three studies have compared the simultaneous use of MMC and AMT in trabeculectomy. In 2008, Sheha and colleagues performed a randomized trial in 37 eyes with refractory glaucoma, comparing trabeculectomy with MMC and single-layer AMT under the scleral flap and trabeculectomy with MMC alone. Twelve months after surgery, results showed that AMT was associated with higher success rates, lower postoperative mean IOP and less complication rates in that group of patients. ${ }^{12}$ In 2010, Mahdy and others studied 30 eyes with pediatric glaucoma divided into two groups: trabeculectomy with MMC and AMT, and trabeculectomy with MMC alone. After 18 months of follow-up, there was no significant difference in the IOP-lowering effect but the group with AMT showed fewer complications. ${ }^{14}$ In 2017, Yadava et al randomized 40 eyes with uncontrolled glaucoma (37 of them with PACG) into two groups: control group underwent trabeculectomy augmented with MMC while the study group underwent additional AMT. At oneyear follow-up, the latter achieved higher rates of complete success in IOP reduction and lesser interventions such as bleb needling. ${ }^{13}$

We conducted this retrospective comparative study in order to further analyze the simultaneous use of amniotic membrane and MMC. Our study is the first comparative study using MSS Trab. Overall, the good results in terms of IOP control and complications are according to the outcomes of this type of surgery.

However, the results did not prove a favorable effect of simultaneous use of MMC and AMT. We did not find significantly different postoperative IOP values, and surprisingly there was a higher need for antihypertensive medications in the AMT group. The need for surgical reinterventions reflected the existence of nonfunctioning blebs, whether they were flat or cystic. In the twelvemonth period, the percentage of eyes that required surgical reinterventions was similar in both groups, as well as the timing of those procedures.

The aforementioned results could be limited by the retrospective nature of the investigation, by the limited sample size, the unequal number of eyes in both study groups and by the heterogeneity of glaucoma types among them. We must point the possible bias of the higher number of antihypertensive medications used in the AMT group. However, tendentiously higher levels of IOP encountered in this group could explain this difference. Besides, the authors performed AMT using one single layer of amniotic membrane in contrast to several previous reports of folded or double layer grafts, which could eventually lead to different outcomes.

Search for new alternatives for fibrosis control is still a challenge in glaucoma surgery. Amniotic membrane could be further studied whether used as a folded graft or in combination with other antifibrotic adjuncts.

\section{Conclusion}

Despite the known antifibrotic properties of amniotic membrane, in our study its addition to MMC-augmented trabeculectomy did not improve surgical outcomes in terms of IOP and need for medications or surgical reinterventions. Further investigations with prospective clinical trials are needed to better understand the role of amniotic membrane in glaucoma surgery. On the other hand, the search for new alternatives to antimitotic drugs is still imperative.

\section{Disclosure}

The authors report no conflicts of interest in this work.

\section{References}

1. Higginbotham EJ, Alexis D. Is newer necessarily better? The evolution of incisional glaucoma surgery over the last 100 years. $\mathrm{Am}$ J Ophthalmol. 2018;191:xxv-xxix. doi:10.1016/j.ajo.2018.04.009

2. Cairns JE. Trabeculectomy. Preliminary report of a new method. Am J Ophthalmol. 1968;66:673-679. doi:10.1016/0002-9394(68)91288-9

3. Khaw PT, Chiang M, Shah P, Sii F, Lockwood A, Khalili A. Enhanced trabeculectomy: the Moorfields safer surgery system. Dev Ophthalmol. 2017;59:15-35.

4. Yoon PS, Singh K. Update on antifibrotic use in glaucoma surgery, including use in trabeculectomy and glaucoma drainage implants and combined cataract and glaucoma surgery. Curr Opin Ophthalmol. 2004;15:141-146. doi:10.1097/00055735-200404000-00015 
5. Slabaugh M, Salim S. Use of anti-VEGF agents in glaucoma surgery. J Ophthalmol. 2017;2017:1645269. doi:10.1155/2017/1645269

6. Akkan JU, Cilsim S. Role of subconjunctival bevacizumab as an adjuvant to primary trabeculectomy: a prospective randomized comparative 1-year follow-up study. $J$ Glaucoma. 2015;24:1-8. doi:10.1097/IJG.0b013e318287abf3

7. He M, Wang W, Zhang X, Huang W. Ologen implant versus mitomycin $\mathrm{C}$ for trabeculectomy: a systematic review and meta-analysis. PLoS One. 2014;9:e85782. doi:10.1371/journal.pone.0085782

8. Fujishima H, Shimazaki J, Shinozaki N, Tsubota K. Trabeculectomy with the use of amniotic membrane for uncontrollable glaucoma. Ophthalmic Surg Lasers. 1998;29:428-431. doi:10.3928/1542-887719980501-12

9. Ji QS, Qi B, Liu L, et al. Comparison of trabeculectomy and trabeculectomy with amniotic membrane transplantation in the same patient with bilateral glaucoma. Int J Ophthalmol. 2013;6:448-451. doi:10.3980/j.issn.2222-3959.2013.04.07

10. Khairy HA, Elsawy MF. Trabeculectomy with mitomycin-c versus trabeculectomy with amniotic membrane transplant: a medium-term randomized, controlled trial. $J$ Glaucoma. 2015;24:556-559. doi:10.1097/IJG.0000000000000060

11. Stavrakas P, Georgopoulos G, Milia M, et al. The use of amniotic membrane in trabeculectomy for the treatment of primary open-angle glaucoma: a prospective study. Clin Ophthalmol. 2012;6:205-212. doi:10.2147/OPTH.S27187
12. Sheha H, Kheirkhah A, Taha H. Amniotic membrane transplantation in trabeculectomy with mitomycin $\mathrm{C}$ for refractory glaucoma. J Glaucoma. 2008;17:303-307. doi:10.1097/IJG.0b013e31815c3a47

13. Yadava U, Jaisingh K, Dangda S, Thacker P, Singh K, Goel Y. Simultaneous use of amniotic membrane and Mitomycin $\mathrm{C}$ in trabeculectomy for primary glaucoma. Indian $J$ Ophthalmol. 2017;65:1151-1155. doi:10.4103/ijo.IJO_447_17

14. Mahdy RA, Nada WM, Almasalamy SM, Anany HA, Almasary AM. A freeze-dried (lyophilized) amniotic membrane transplantation with mitomycin C and trabeculectomy for pediatric glaucoma. Cutan Ocul Toxicol. 2010;29:164-170. doi:10.3109/15569521003775005

15. Dua HS, Gomes JA, King AJ, Maharajan VS. The amniotic membrane in ophthalmology. Surv Ophthalmol. 2004;49:51-77. doi:10.1016/j.survophthal.2003.10.004

16. Zhong Y, Zhou Y, Wang K. [Effect of amniotic membrane on filtering bleb after trabeculectomy in rabbit eyes]. Yan Ke Xue Bao. 2000;16 (73):83. Chinese.

17. Wang L, Liu X, Zhang P, Lin J. [An experimental trial of glaucoma filtering surgery with amniotic membrane]. Yan Ke Xue Bao. 2005;21:126-131. Chinese.

18. Eliezer RN, Kasahara N, Caixeta-Umbelino C, Pinheiro RK, Mandia C Jr, Malta RF. Use of amniotic membrane in trabeculectomy for the treatment of glaucoma: a pilot study. Arq Bras Oftalmol. 2006;69:309-312. doi:10.1590/S0004-27492006000300005
Clinical Ophthalmology

\section{Publish your work in this journal}

Clinical Ophthalmology is an international, peer-reviewed journal covering all subspecialties within ophthalmology. Key topics include: Optometry; Visual science; Pharmacology and drug therapy in eye diseases; Basic Sciences; Primary and Secondary eye care; Patient Safety and Quality of Care Improvements. This journal is indexed on PubMed

\section{Dovepress}

Central and CAS, and is the official journal of The Society of Clinical Ophthalmology (SCO). The manuscript management system is completely online and includes a very quick and fair peer-review system, which is all easy to use. Visit http://www.dovepress.com/ testimonials.php to read real quotes from published authors. 